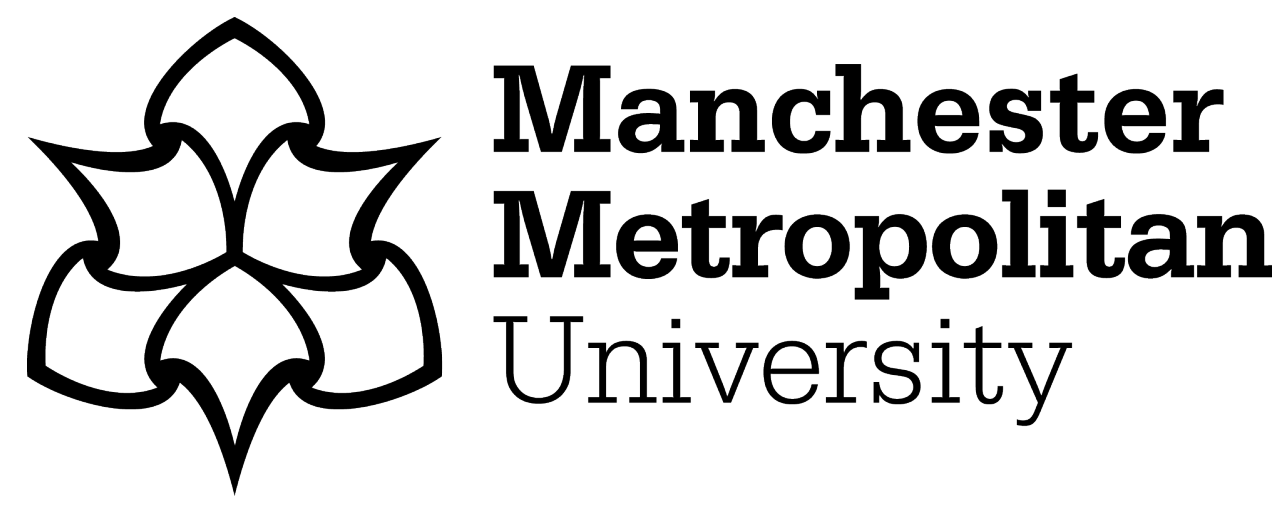

Woolley, Martin and Niedderer, Kristina (2016) Editorial: Real or unreal? Crafting authenticity in the digital age. Craft Research, 7 (2). pp. 159-164. ISSN 2040-4689

Downloaded from: https://e-space.mmu.ac.uk/622130/

Publisher: Intellect

DOI: https://doi.org/10.1386/crre.7.2.159_2

Please cite the published version 


\title{
EDITORIAL
}

\author{
Martin Woolley \\ Coventry University, UK
}

Kristina Niedderer
University of Wolverhampton

\section{Real or unreal? - Crafting authenticity in the digital age}

This special issue focuses on the meaning and relevance of authenticity of craft in the digital age. There are a number of concerns that are important in the discussion of authenticity. Firstly, current developments, including computer aided manufacturing and science-based ways of 'producing' craft artefacts, such as growing clothing from micro cultures, raise the need to question established understandings of making and of craft in terms of the hand-made and its individuality. Secondly, continuing developments of globalisation, mass production, and the overlap of craft and design domains there raise questions about use, development and ownership of traditional and new designs. Thirdly, authenticity is regularly related to issues of originality, representation, and stewardship, because in relation to hand-made artefacts, authenticity is often connected with authentication. As in any financial transaction, authentication is viewed as the responsibility of the vendor to demonstrate that the object is the genuine article it purports to be. In the case of heritage objects, antiques for example, expert verification of the origins of the objects dominates most transactions. In terms of contemporary craft, the position is more ambiguous. Perceptions of what is or should be regarded as 'genuine' are being blurred as a consequence of changes in technologies and working practices, including e.g. digital manufacture or the growing of 'bio-fashion', which each embed their own understandings and values in the work.

Part of the attraction of craft objects can derive from an understanding of how they are made, what they are made of, an appreciation of the skill required to produce them and from '... understand[ing] and enjoy[ing] the energy and care which has gone into their making' (Greenlees 2014). Such shared knowledge between maker and audience can contribute to a form of authentication, evidenced through visual characteristics of the object which signal its origins. Visible traces of the maker's skills and associated variation between individual pieces through making by hand, even where producing repeat patterns, are therefore traditionally seen as a central characteristic of craft. With the rise of digital and science driven manufacture, the question arises as to where the signature of the maker might reside within mass customisation, now that wide variation and individualisation can be produced at the push of a button or in the 'petri dish'.

This reopens the question as to how the hand signifies making and what its role is in relation to design, referring to the link between creativity, thinking and the hand. In this special issue, several authors consider authenticity in relation to their own practice, with Kettley tackling the issue by exploring a decade of her own digital practice in an effort to identify the wider relationship between craft and authenticity. She argues that it is deeply embedded in craft thinking and goes on to propose a new model through which craft's authenticity can be interrogated. Loh also explores digital technology but in relation to the production process, with specific reference to Computer Numeric Control (CNC) making in the context of architectural model-making. 
A similar question concerning authenticity arises in the context of globalisation, albeit from a different starting point: with established craft practices, especially where they are exercised in traditional settings, today it can be hard for crafts people to maintain their practices, because of poor pay, lack of recognition or lack of materials. Perceptions of the need for improvement, sometimes government driven, sometimes community driven, can lead to interventions that raise questions about the ownership of the craft work, both for the makers as well as others involved in the interventions, such as designers. This is because traditionally, craft has evolved incrementally around a specific style, which was 'community owned' and which was improvised upon by the practitioners. In a modern world, this work ethic clashes with the 'idolisation of designers (or artists), who gain individual recognition (and money), while crafts people remain anonymous and hence often underpaid. Salim discusses just such scenarios with regard to the clay traditions in Sindh, a province in Southern Pakistan. Salim compares several case studies on this issue to examine different collaborative practices and interventions in relation to their effectiveness and benefit for the makers.

The Industry Report in this issue also takes specific craft materials as a starting point with Soylu and ER focussing their investigation on needlecraft, with reference to a global supplier of sewing and embroidery threads. Whilst not addressing authenticity directly, it is clear from the combined findings of the company case study and extensive market research data, that psychological rather than physical needs predominate as the socio-economic status of the consumer increases. Potentially at least, authenticity in the form of indicators of quality and genuineness may help to support self-realization as defined by Maslow's 'hierarchy of needs' (Maslow 1943), particularly in these higher socioeconomic groups.

Authenticity in relation to representation has emerged as a third issue, raising questions such as 'How can we authenticate the digital and how might makers address genuineness, the ownership of ideas, designs and claims to uniqueness, in a world of instant copying, sampling and the habitual plagiarism of images?' In the light of such developments, one might also question what the meaning of authenticity is, whether it has changed and how, and also how important authenticity is in the digital age in relation to the cult of originality, and the manipulation of existing designs. By extension, one can further speculate whether the tradition of the developing body of personal work, which has long functioned as a key indicator of authenticity, will continue in the face of rapidly mutating, technological opportunities, and what might replace it. We already speak of 'hybrid craft' but what does it mean, and what does it imply about the future of craft?

In her position paper, Myzelev looks at authenticity and hybrid craft in relation to representation in the context of three different exhibitions, where she considers the presentation of craft in different contexts and its representation through traditional artistic media, such as photography, and new digital platforms such as online exhibitions. She highlights the difficulty of presentation and interpretation, even of original artefacts, which can lead to misrepresentation, dependent on which perspective one takes. Led by these insights, she argues that in the digital age, authenticity is becoming blurred to such an extent as to loose its relevance. Taking a focus on representation of identity from the maker's perspective, White examines authenticity in relation to a specific example of portraiture where she reworked an image based on an early police mug shot. This was in the form of an embroidered portrait which sought to reveal a more rounded and nuanced 'truth' about the life and person behind the image, than the stark functionality of the original photograph would allow.

The maker's review focuses on the life and work of Eleanor Glover and explores authenticity in relation to the influence of lived experience on her mixed media sculptures and animations. The emergence of ubiquitous maker websites has facilitated the mass distribution of biographical 
information, photographs, and video clips, alongside craft images. Glover is typical of this development in exploiting the web and social media to outline her background and create a continuing contextual narrative intrinsic to her work, partly as a means of demonstrating authenticity. It can be argued that as a first point of contact, maker web-sites tend to present a pre-purchase narrative, as opposed to a previous era when in the 1990's non-digital narratives were the order of the day and tended to function as a post-purchase narrative only. 'A craftsperson's resume, biography or point-ofpurchase literature is usually consulted after a selection has been made. Information about the maker is used to confirm, not motivate, the purchase. It may be retained to show others as validation, and some crafts-people issue 'certificates of authenticity.' (Hickey 1997)

The significance of the maker's story in relation to practice is also explored by Campbell of the Crafts Council in her review of 'Making Movies', the first UK craft film festival. She is clear that 'within a contemporary culture of effortless access to increasingly ubiquitous and hi-spec technology, we are seeing contemporary makers progressively explore and exploit the opportunities it offers and integrating moving-images into their broader working practice.' Interestingly she also argues that many of the films are art works in their own right and not merely a backdrop to, or authentication of, practice itself.

It is equally possible that authenticity is becoming the new consumer sensibility; the buying archetype by which consumers are choosing what and who they wish to form an economic relationship with and why (Devers 2012). In the industrial era it became easy to present a deceptive surface authenticity that bore little relationship to the below-the-line reality of the object. Modern digital production techniques have simply extended this option to the level of unique rather than volume produced objects. Equally there are those who would question the entire relevance of authenticity and craft in general and are '.. witheringly critical about those who get this wrong by making a fetish of craft itself, because of a misplaced love of its archaism or authenticity.' (Adamson 2013). Clearly the debates surrounding authenticity are both broad and challenging and it is hoped that this journal issue will go some way to air and focus some of the key arguments.

\section{A word of thanks}

We are delighted to present Volume 7.2 of Craft Research. As always, many people have been involved in the realisation of this issue. We wish to thank all our contributors, as well as those authors whose submissions we regrettably had to turn away. Our gratitude also extends to all our advisors as well as to our reviewers for their excellent work. Their constructive advice and feedback to authors is an essential part in fulfilling the developmental role of the journal and in advancing the field. We further wish to thank Intellect Publishers for their continued support for the journal, in particular our journal's manager, Bethan Ball, and her team.

Martin Woolley and Kristina Niedderer

September 2016 


\section{References}

Greenlees, R. (2014), 'What is Craft? - Definitions of craft', V \& A Crafts.

http://www.vam.ac.uk/collections/contemporary/crafts/what is craft/index.html. Accessed 15th August 2016.

Maslow, A. H. (1943), A theory of human motivation, Psychological Review, 50 (4), p. 370.

Hickey, G. (1997), Craft within a consuming Society, In: Dormer, P. (ed.) The Culture of Craft, p.86

Devers, E., (2012), The Art of Craft and Valuing Authenticity | Design Online [WWW Document]. Design Online, State Library of Queensland, Australia. URL http://designonline.org.au/content/theart-of-craft-and-valuing-authenticity/ (accessed 8.15.16).

Adamson, G. (2013), Thinking Through Craft. Bloomsbury, London, P.11

\section{Contributor details}

Professor Martin Woolley is Emeritus Professor in Design Research, Faculty of Arts and Humanities, Coventry University. With an early background in three-dimensional and industrial design, his research interests have broadened to encompass technology transfer, new product development, the crafts, environmental sustainability and user-centred design. He has supervised and examined numerous research degrees, and has been active in several national and international research bodies and on UK government research panels. He has also acted as principal investigator on several major funded research projects, and has published widely.

Contact:

Faculty of Arts and Humanities, Coventry University, Priory Street, Coventry, CV1 5FB, UK

Email: martin.woolley2@coventry.ac.uk

Kristina Niedderer, Ph.D. M.A. (RCA), is Professor of Design and Craft at the University of Wolverhampton. She was originally apprenticed and worked as a goldsmith and silversmith in Germany. She then trained as a designer and design researcher in the United Kingdom, with an M.A. (RCA) and a Ph.D. in Design. Niedderer's research focuses on the impact of design on human relationships, investigating the role of mindfulness and emotions as a means to engender mindful interaction and behaviour change through design. Niedderer is a Council Member and Secretary for Special Interest Groups of the Design Research Society and she leads the European project 'Designing for People with Dementia', Marie Skłodowska-Curie grant agreement No 691001.

Contact:

Faculty of Arts, University of Wolverhampton, Molineux Street, Wolverhampton, WV1 1DT.

E-mail: k.niedderer@wlv.ac.uk

Web address: http://www.niedderer.org 